\title{
Cytomegalovirus infections in pregnant women as a risk of congenital deafness in a child
}

\author{
Wiesław Konopka, Małgorzata Śmiechura-Gańczarczyk, Renata Pepaś \\ Department of Otolaryngology, Polish Mother's Memorial Hospital-Research Institute, Lodz, Poland
}

\begin{abstract}
Introduction: One per cent of live births are affected by cytomegalovirus infection, but $90 \%$ of neonates with perinatal infection do not show symptoms of disease. Symptomatic cytomegalovirus (CMV) is present in $5-10 \%$ of children. Typical clinical signs of congenital cytomegalovirus infection are microcephalia, mental retardation, progressive major amblyacousia, and neuromuscular infection. Hypoacusis is present in $30-60 \%$ of children with congenital symptomatic CMV - in most cases it is bilateral and applies to high-frequency hearing loss.

Material and methods: A group of 70 children had serological and genetic screening of viral DNA using the polymerase chain reaction method in urine and blood. In this group, 52 children were diagnosed with congenital CMV, and 10 children were diagnosed with acquired CMV. Audiological examinations including pure-tone audiometry, auditory brainstem response, transiently evoked otoacoustic emission, and immittance audiometry were performed.

Results: Bilateral sensorineural hearing loss was in 9 children associated with mental and physical retardation, brain malformation, and microcephalia. Unilateral hearing loss was observed in 3 children. In 40 cases, we did not observe hearing loss, although the level of bilirubin was high, as well as splenomegaly, hepatomegaly, and facial nerve paralysis.

Conclusions: Congenital cytomegalovirus infection often caused hearing loss. Children with congenital and acquired CMV should be under the permanent care of an audiologist. The growing problem of CMV infections and their late diagnosis suggests the need to prepare screening tests and increase the education of gynaecologists, neonatologists, paediatricians, and general practice doctors about CMV problems.
\end{abstract}

Key words: pregnancy, CMV, children, hearing loss.

\section{Introduction}

At the beginning of the $20^{\text {th }}$ century, congenital cytomegalovirus infection was described as an insertion disease due to the discovery of large cells with insertions inside the nucleus and cytoplasm in the necropsy material taken from saliva glands. Weller introduced the name "cytomegalovirus" in 1960 based on histopathological examination [1]. Cytomegalovirus is a herpes virus with double-stranded DNA, the nucleocapsid of which is surrounded by a lipid-protein layer. The congenital cytomegalovirus infection (CMV) is one of the most common intrauterine diseases. The cytomegalovirus infection affects $40-100 \%$ of the population, and it is more frequent in developing countries and among people of lower economic status [2]. In Poland, the percentage of seropositive patients can be estimated at $70 \%$ of the population [3].

It is estimated that the disease can affect 0.64-0\%, and $70 \%$ of newborn children in the world [4]. Sensorineural hearing loss (SNHL) is the most common sequela of congenital CMV infection, occurring in $10-15 \%$ of all infected children. Hearing loss reportedly occurs in $30-40 \%$ of children who have clinically apparent disease (symptomatic) at birth and in 5-10\% of children with clinically silent (asymptomatic) infections. In the USA, $15-21 \%$ of all congenital hearing loss can be caused by this infection $[5,6]$.

The multiplication of viruses can cause active infection with the whole range of clinical effects or can have no side effects and transfer into latency. When the infection affects people with an efficient immune system it is most commonly asymptomatic or manifests with mild, mononucleosis-like symptoms. The CMV infections are divided into primary and secondary types. The primary type can be characterized by the immunoglobulin (Ig) M anti-CMV antibodies found in the blood serum of previously seronegative patients. The secondary type involves reactivation of hidden infection or a secondary infection caused by another virus type in a person with anti-CMV antibodies. The direct impact 
Table 1. Average pure-tone audiogram congenital cytomegalovirus

\begin{tabular}{lcccccccccc}
\hline Frequency & $\mathbf{k H z}$ & $\mathbf{0 . 1 2 5}$ & $\mathbf{0 . 2 5}$ & $\mathbf{0 . 5}$ & $\mathbf{1}$ & $\mathbf{2}$ & $\mathbf{3}$ & $\mathbf{4}$ & $\mathbf{6}$ & $\mathbf{8}$ \\
\hline Right ear $n=10$ & $\mathrm{~dB}$ & 75 & 80 & 75 & 80 & 85 & 90 & 90 & 95 & 90 \\
\hline Left ear $n=11$ & $\mathrm{~dB}$ & 75 & 75 & 75 & 85 & 80 & 95 & 90 & 95 & 95 \\
\hline $\mathrm{SD} \pm$ & $\mathrm{dB}$ & 4.0 & 3.0 & 2.0 & 5.0 & 5.0 & 4.5 & 3.0 & 3.0 & 4.0 \\
\hline
\end{tabular}

of the virus on the cell is not the only key factor in the mechanism of changes - the typical inflammatory reaction of blood vessels with leukocyte infiltration is also very important.

It is estimated that symptomatic CMV is in $90 \%$ associated with the risk of ophthalmic and audiological damage of varied intensity. However, in children with delitescent infection there is a $10-15 \%$ risk of hearing loss [2, 7-10].

The purpose of this thesis is the evaluation of hearing in children with congenital and acquired cytomegalovirus infection.

\section{Material and methods}

The research was conducted on a group of 70 children (39 boys and 31 girls) at the age of 2 to 12 years (average age: 6 years) with diagnosed congenital and acquired cytomegalovirus infection; audiological examinations were performed.

The cytomegalovirus infection was diagnosed based on clinical records and serological and molecular screening. The characteristic anti-CMV antibodies were marked by the immune-enzymatic method called ELISA. The presence of the virus DNA (CMV DNA) in urine and blood was checked using polymerase chain reaction (PCR).

All children went through a full laryngological examination in order to exclude ear, nose, and throat anomalies. The next step was an audiological examination of hearing to define the gradation and localization of hearing loss.

The hearing examination was conducted on 70 children. Audiological examination consisted of pure-tone audiometry (PTA) (with the use of a Madsen 622 audiometer and TDH 39 headphones) for air conduction at the frequency of $125-8000 \mathrm{~Hz}$ and bone conduction at the frequency of $250-4000 \mathrm{~Hz}$. The impedance audiometry test (Zodiac 901P immittance audiometer, Madsen) was conducted by recording the tympanogram and the reflex of the stapes muscle. The ipsilateral and contralateral stimulation was performed at $226 \mathrm{HZ}$ of the probe trial tone. The recording of transiently evoked otoacoustic emission (TEOAE) was performed using an Echocheck probe, and the answers from the brain stem (ang. auditory brainstem response - ABR) were examined with the use of a Racia device; the evoked response from a click was repeated 1600 times (one patient was in a pharmacological coma).
Additionally, all parents were thoroughly interviewed regarding the course of pregnancy, childbirth, and infancy and the current health condition of their children.

The research was carried out in accordance with the Helsinki Declaration.

\section{Results}

Congenital cytomegalovirus infection was diagnosed in 52 children and acquired CMV in 18 children.

Nine children ( 5 girls and 4 boys - above 3 years old) among the patients with congenital CMV (17\% of the group) were diagnosed with serious bilateral SNHL (pure-tone average at 85-90 dB) and 3 had unilateral hearing loss -2 left ear and 1 right. The pure-tone audiometry of all examined children showed similar air and bone conduction at low and middle frequencies at the level of $70 \mathrm{~dB}$, while at the frequency above $2000 \mathrm{~Hz}$ the level was $90 \mathrm{~dB}$ (Table 1).

The auditory brain stem response (ABR) of 9 children from this group with serious hearing loss did not show repetitive response to an $80 \mathrm{~dB}$ sound stimulus in both ears. The $V$ wave was present bilaterally with a $90 \mathrm{~dB}$ stimulus. No TEOAE was recorded in this group. The $A B R$ test of 2 children did not show repetitive answers to the $100 \mathrm{~dB}$ click, and 1 (child) did not show repetitive answers at $40 \mathrm{~dB}$. No otoacoustic emission (TEOAE) was recorded. In 1 of 2 children without response at $100 \mathrm{~dB}$ by using $A B R$, during the one-year period we observed progressive hearing loss from the normal hearing level.

Immittance audiometry performed in this group showed the presence of a type A tympanogram and a lack of stapes muscle reflex both with ipsilateral and contralateral stimulation.

Normal hearing was recorded in 40 (57\%) children (17 girls and 23 boys) from the group with congenital CMV, average PTA 15-20 dB HL and the ABR test - the $\checkmark$ wave was present with a $20 \mathrm{~dB}$ stimulus, with positive results of immittance audiometry and positive TEOAE. The children from this group additionally suffered from hyperbilirubinaemia $(18,21 \mathrm{mg} / \mathrm{dL})$, liver and spleen enlargement, and unilateral facial nerve paralysis (2 children). Only 1 child was not diagnosed with malfunction of other organs.

In the group of 9 children with bilateral serious SNHL, CMV was diagnosed in infancy as well as mental and physical retardation, enlargement of the ventricular system, and microcephaly. Children with unilateral hearing loss had no additional abnormalities. 
In the group of 18 children with acquired CMV (8 girls and 10 boys) the hearing threshold was normal $(10-20 \mathrm{~dB})$, as confirmed by the PTA, the appropriate results of impedance audiometry, the presence of the evoked otoacoustic emission (TEOAE), and hearing threshold in the ABR test at the level of 15-20 dB.

Mental and physical retardation was diagnosed in 2 children, but their hearing was normal.

\section{Discussion}

Humans are the only reservoir of CMV, and infections can spread horizontally or vertically. The source of infection can come from body fluids such as urine, saliva, breast milk, blood, and transplanted organs. Vertical CMV infection spread can occur in utero, as well as during and after childbirth. Severe infections concern people with immunodeficiency as well as newborns and infants infected in utero at various stages of pregnancy, as a result of primary maternal infection or reactivation of latent infection. It is estimated that in pregnant women, in the case of primary infection, the risk of transmission of the infection to the foetus is $24-75 \%$ (average $50 \%$ ), and in secondary infections it is approximately $1 \%$. Young mothers with low economic status who have contact with young children are at a higher risk of primary infection. Clinical signs of CMV disease at birth are seen in only $10 \%$ of infected newborns [11, 12].

Without a screening program for CMV infections in pregnant women, a high percentage of asymptomatic infections in newborns and uncharacteristic clinical signs of infection complicate the early diagnosis of cCMV. The diagnosis of congenital cytomegalovirus infection is possible in utero in children with diagnosed abnormalities in the ultrasound examination. This method, due to its wide availability, allows diagnosis of microcephaly, dilatation of the ventricular system, calcifications, and intracerebral bleeding. The risk of CMV infection is increased in children of mothers with confirmed seroconversion of anti-CMV IgM/lgG antibodies during pregnancy; however, the serological status of the contraceptive period is sporadically known. It was also found that the presence of anti-CMV antibodies does not significantly correlate with the presence of CMV-DNA in the mother's blood, and the presence of CMV genetic material in the plasma of pregnant women is not synonymous with foetal infection. Therefore, the risk of transmission of CMV infection to the foetus should be assessed based on PCR testing of amniotic fluid with amniocentesis after 20 weeks' gestation. CMV can be identified in the amniotic fluid more than 6 weeks after maternal signs of infection. An analysis of the studies carried out by Guerra et al. allowed the establishment of a threshold of 105 CMV copies in $1 \mathrm{ml}$ of amniotic fluid, above which symptomatic infections of newborns are observed [13].
Due to the possibility of acquired CMV infection in early infancy, it is necessary to establish the diagnosis of congenital infection by the 21st day of life of the newborn. Diagnosis of CMV is recommended by amplification of viral DNA in urine, saliva, or CSF by PCR. The latest research by Boppana et al. confirms the high sensitivity and specificity of the PCR tests of liquid and dried saliva, which makes it possible to develop newborn screening tests based on diagnostic material which is easy to collect and store. It is well known that the presence of IgM antibodies in a baby after birth can indicate a congenital infection. However, due to an insufficient immune response of newborns, only $70 \%$ of those infected with CMV have IgM antibodies. Diagnostic tests are also characterized by different sensitivity and specificity, which means that CMV diagnosis based only on serological tests is not recommended [14, 15].

Hearing loss is caused by CMV infection in $10-15 \%$ of infected children (it applies to $30-40 \%$ of the diagnosed patients with symptomatic infections and 5-10\% with asymptomatic ones) [8, 16-18]. Hearing loss caused by the CMV can be both bilateral and unilateral, with symptoms detected at the moment of birth or during the first year of life. The gradation of hearing loss can differ from medium to high, and it can be fluctuating or gradually decreasing. Therefore, up to half of CMV cases are not diagnosed during infant hearing tests [19].

Dollard et al. observed SNHL hearing loss in $41 \%$ of a group with symptomatic infections and in $8.7 \%$ of asymptomatic infections $[10,11]$. The first descriptions of SNHL associated with CMV infection were made only 40 years ago. So far, no single cause of SNHL has been discovered $[8,19]$.

The progressive SNHL may affect $60 \%$ of newborns and can lead to deafness in $30 \%$ of newborn children. Cochlear implantation was effective for deaf children with congenital CMV infection, but their cochlear implant outcomes were often impaired, depending on the types of CMV-associated psycho-neurological disorders [20].

Barbi et al. detected congenital CMV infection in $17 \%$ of children with SNHL higher than $40 \mathrm{~dB}$. This group consisted of $10 \%$ of children with hearing loss detected shortly after birth and $30 \%$ of children with unknown aetiology diagnosed 3 months after birth [21]. Ogawa et al. diagnosed congenital CMV infection in $15 \%$ of 67 children examined due to high sensorineural hearing loss. In the group of 55 children with medium to high hearing loss (> $55 \mathrm{~dB}$ ) $16 \%$ of children had congenital CMV infection, and in the group of 36 children with high bilateral sensorineural hearing loss, $22 \%$ were diagnosed with congenital CMV [22]. According to other authors, 30-50\% of children with CMV symptoms had sensorineural hearing loss, while asymptomatic infection concerned only $8-12 \%$ of children $[5,6,17]$. In our material, the percentage of SNHL in asymptomatic infection was $17 \%$. The latest research indicates that 
SNHL is diagnosed in $21 \%$ of newborn children and in $25 \%$ of 4 -year-olds. The data suggest that hearing loss does not have a genetic background $[5,6,17]$. Most children with congenital CMV infection do not develop hearing loss; however, it is unclear which children with congenital CMV infection will develop hearing loss and, among those who do develop loss, whether or not the loss will continue to deteriorate. In our material, this was $35 \%$ of the examined group. Physical retardation caused by the damage of the central nervous system and other organs can be diagnosed in children born without clinical symptoms. In our material, such a case applied to 1 child.

Studies of human temporal bones along with studies of animal models indicate that CMV (or a CMV antigen) may be present in the epithelium and the neural cells in the inner ear. CMV may cause damage to the inner ear by virus-mediated damage to the neural cells and/or secondary to host-derived inflammatory responses to CMV in the ear resulting in injury to the auditory apparatus and subsequent hearing loss. However, these explanations do not fully explain the progressive and late-onset nature of CMV-related hearing loss. The pathogenesis of hearing loss caused by congenital CMV infection is not well understood; however, animal models and studies of temporal bone structures from infants with severe congenital infection suggest some hypotheses [23].

Acquired CMV infection is most often asymptomatic; however, its course can take the form of mononucleosis-like symptoms or cytomegalovirus liver infection $[20,24]$. Post-transfusion cytomegalovirus infection is most often diagnosed in prematurely born sick children who have undergone numerous blood transfusions, and it is characterized by septic symptoms, liver infection, and hepatosplenomegaly, pneumonia without fever, haemolytic anaemia, atypical lymphocytosis, thrombocytopaenia, and neutropaenia. The mortality rate in this group amounts to $20 \%$. Patients with a well-functioning immune system infected with cytomegalovirus undergo symptomatic medical treatment. Patients with immune deficiency and cytomegalovirus infection should be treated with ganciclovir, a synthetic analogue of 2'-deoxy-guanosine. However, this treatment - especially in the case of newborn children - is controversial due to ganciclovir's high toxicity. Ganciclovir inhibits CMV DNA polymerase. Ganciclovir is infused every 12 hours for 14-21 days. The duration of treatment depends on the clinical record of infection and the tolerance to the medication [25]. Research done by clinics in the USA in recent years indicate that ganciclovir treatment in newborn children with congenital symptomatic cytomegalovirus lasting 6 weeks reduces the risk of hearing impairment; however, it increases the risk of neutropaenia occurrence. With reference to the above, it is necessary to monitor the functioning of kidneys and haematological parameters. The results obtained in the above-mentioned examination confirm the purpose of the ganciclovir treatment in prophylaxis of hearing loss caused by CMV [26]. Hyperimmunized immunoglobulin or any standard gamma globulin can be used as a supporting treatment (in conjunction with ganciclovir). Other medications used in CMV infections include Foscarnet, among others. However, $10-23 \%$ of patients suffer from its nephrotoxic effect; therefore, its usage is limited in patients after kidney transplantation. Due to an increasing number of reports on CMV strains resistant to the currently used drugs, research has been carried out to find new potent drug derivatives such as Cidofovir, Lubocovir, or Valganciclovir. The active immunoprophylaxis still faces difficulties related to latency and - most probably - the oncogenic qualities of the virus. Nowadays, various vaccines against the cytomegalovirus are being developed and tested: live attenuated vaccine, peptide vaccine, and the most recent with live genetically modified viruses. However, due to numerous mutations of the virus and the superinfections caused by a new strain despite the immunization, there is a risk of a recurring infection. Therefore, prophylactic measures, such as appropriate hygienic behaviour and avoiding multiple sexual partners by women in their reproductive years, remain very important [24].

Because CMV-related hearing loss may be late-onset and/or progressive, infants with congenital CMV infection should have early and more frequent audiological evaluations to assess for possible changes in their hearing status, so that appropriate interventions may be implemented to minimize the impact of their hearing loss. Unstable hearing thresholds are frequently found in children with congenital CMV infection and occur not only in children who develop hearing loss but also in children who have normal hearing. There are data that CMV-related hearing loss is associated with ongoing viral replication in the inner ear even for years after birth [27-29].

Approximately $50 \%$ of children with SNHL due to congenital CMV infection will have progressive hearing loss. In our material, this concerned 1 child. Children with congenital CMV infection should have hearing tests every 6 months until the end of the speech development period, to monitor for possible changes to their hearing loss. However, at times when hearing loss appears to be changing, audiological evaluations may be needed every 3 months to assess and document the changes in the hearing status of the child infected with CMV.

\section{Conclusions}

Hearing loss was diagnosed only in the group of children with congenital cytomegalovirus.

Most patients with congenital cytomegalovirus suffered from serious malfunctioning of other organs. 
Frequent and routine audiological follow-up is needed to identify children with CMV-related hearing loss.

Early identification of congenital CMV infections allows qualification for antiviral therapy and thus reduces the risk of complications.

\section{Disclosure}

The authors report no conflict of interest.

\section{References}

1. Weller T, Hanshow J, Scott JB. Serologic differentiation of viruses responsible for cytomegalic inclusion disease. Virol 1960; 12: 130-132.

2. Conboy TJ., Pass RF. Stagnos and partners: intellectual development in school-aged children with asymptomatic congenital cytomegalovirus infection. Pediatrics 1986; 77: 801-806

3. Woźniakowska-Gęsicka T, Wróblewska W, Wiśniewska-Ligier M. Rola wirusa cytomegalii w patologii wieku rozwojowego. Ped Pol 2006; 81: 674-678.

4. Kenneson A, Cannon MJ. Review and meta-analysis of the epidemiology of congenital cytomegalovirus (CMV) infection. Revi Med Virol 2007; 17: 253-276.

5. Grosse SD, Dollard SC, Ross DS. Congenital cytomegalovirus (CMV) infection as a cause of permanent bilateral hearing loss: a quantitative assessment. J Clin Virol 2008; 41: 57-62.

6. Morton CC, Nance WE. Newborn hearing screening - a silent revolution. NEngl J Med 2006; 354: 2151-2164.

7. Boppana SB, Pass RF, Britt WJ, et al. Symptomatic congenital cytomegalovirus infection in infants born of mothers with preexisting immunity to cytomegalovirus. Pediatr Infect Dis J 1992; 11: 93-99.

8. Dahle AJ, Fowler KB, Wright JV, et al. Longitudinal investigation of hearing disorders in children with congenital cytomegalovirus. J Am Audiol 2000; 5: 283-290.

9. Demmler GJ. Infections diseases Society of America and centers for disease control. Rev Inf Dis J 1991; 13: 315-329.

10. Dent KM, Kenneson A, Palumileos JC. Methodology of multistate study of congenital hearing loss preliminary date from Utah newborns screening. Am J Med Genet 2004; 15: 28-34.

11. Stagno S, Pass RF, Cloud G, et al. Primary cytomegalovirus in pregnancy. JAMA 1986; 256: 1904-1908.

12. Hollier LM, Grissom H. Human herpes viruses in pregnancy: cytomegalovirus, epstein- barr virus and varicella zoster virus. Clin Perinatol 2005; 32: 671-696.

13. Guerra B, Lazzarotto T, Quarta S, et al. Prenatal diagnosis of symptomatic congenital cytomegalo-virus infection. Am J Obstet Gynecol 2000; 183: 476-482.

14. Boppana SB, Ross SA, Novak Z, et al. Dried blood spot real-time polymerase chain reaction assays to screen newborns for congenital cytomegalovirus infection. JAMA 2010; 303: 1375-1382.

15. Boppana SB, Ross SA, Shinamura M. Saliva polymerase chain reaction assay for cytomegalovirus screening in newborns. N Engl J Med 2011; 364: 2111-2118.

16. Ross S, Fowler K, Ashrith G, et al. Hearing loss in children with congenital cytomegalovirus infection born to mothers with preexisting immunity. Pediatrics 2006; 148: 332-336.

17. Fowler KB, Boppana SB. Congenital cytomegalovirus (CMV) infection and hearing deficit. J Clin Virol 2006; 35: 226-231.

18. 18. Dollard SC, Grosse SD, Ross DS. New estimates of the prevalence of neurological and sensory sequelae and mortality associated with congenital cytomegalovirus infection. Rev Med Virol 2007; 17: 355-363.

19. Fowler KB, Dahle AJ, Boppana SB, Pass RF. Newborn hearing screening: will children with hearing loss due to congenital cytomegalovirus infec tion be missed? J Pediatri 1999; 135: 60-64.

20. Yamazaki H, Yamamoto R, Moroto S, et al. Cochlear implantation in children with congenital cytomegalovirus infection accompanied with psycho-neurological disorders. Acta Otolaryngol 2012; 132: 420-427.
21. Barbi M, Binda S, Caroppa S, Amborestti U, Corbetta C, Sergi P. A wider role for congenital cytomegalovirus infection in sensorineural hearing loss. Pediatr Infect Dis J 2003; 22: 39-42.

22. Ogawa $H$, Suzutani $T$, Baba $Y$, et al. Etiology of severe sensorineural hearing loss in children: independent impact of congenital cytomegalovirus infection and GJB2 mutations. J Infect Dis 2007; 195: 782-788.

23. Schleiss MR, Choo DI. Mechanisms of congenital cytomegalovirus-induced deafness. Drug Disc Today Dis Mech 2006; 3: 105-113.

24. Woźniakowska-Gęsicka T, Wróblewska W, Wiśniewska-Ligier M. Rola wirusa cytomegalii w patologii wieku rozwojowego. Ped Pol 2006; 81 674-678.

25. Sławska H, Czuba B, Mazurek U, et al. Pregnant cytomegaloinfection ability of diagnostic methods in perinatal to fetal transmission recognition. Arch Perinat Med 2003; 9: 45-48.

26. Kimberlin DW, Lin CY, Sanchez PJ, et al. Effect of ganciclovir therapy on hearing in symptomatic congenital cytomegalovirus disease involving the central nervous system: a randomized, controlled trial. J Pediatr 2003; 143: 16-25.

27. Vries JJ, Vesseur A, Rotteveel LJ, et al. Cytomegalovirus DNA detection in dried blood spots and perilymphatic fluids from pediatric and adult cochlear implant recipients with prelingual deafness.J Clin Virol 2013; 56: 113-117.

28. Foulon I, Naessens A, Faron G, Foulon W, Jansen AC, Gordts F. Hearing thresholds in children with congenital CMV infection: a prospective study. Int J Pediatr Otolaryngol 2012, 76: 712-717.

29. Kato K, Otake H, Tagaya M, et al. Progresive hearing loss following ac quired cytomegalovirus infection in an immunocompromised child. Am J Otolaryngol 2013; 34: 89-92. 